\title{
Decking out your DNA
}

Certain DNA polymerases show surprising tolerance for chemically modified nucleotides, opening up exciting new possibilities for the efficient production of highly functionalized nucleic acid molecules.

One important benefit of the ongoing nanotech revolution has been the recognition of nucleic acids as a useful and versatile scaffold for building more complex biochemical tools. A variety of specific functional groups can be covalently tacked onto nucleotides, which can in turn be assembled into chains via several chemical reactions. This can be laborious, however, and some scientists are now looking into the possibility of using naturally occurring enzymes as a more efficient alternative.

One such researcher is Michael Famulok, of the University of Bonn, whose group has published several articles demonstrating the feasibility of this approach. More recently, they scaled up their efforts, systematically measuring the incorporation of a variety of nucleotides containing different functional groups - acidic, basic or lipophilic-into oligonucleotide chains using seven different bacterial DNA polymerases. Some enzymes had only a limited ability to work with unorthodox substrates, but two polymerases, Pwo and Vent (exo ${ }^{-}$) stood apart from the rest, efficiently incorporating every nucleotide variant tested. In subsequent experiments, Famulok's team delineated template sequence determinants that may affect the efficiency of incorporation and demonstrated the efficient enzymatic synthesis by these two polymerases of oligonucleotides containing variants of all four nucleotide types, as well as the successful PCR amplification of a modified template to produce a similarly functionalized product.

The flexibility of these polymerases could have dramatic implications for future biochemical engineering projects, such as aptamer development. "Additional chemical functionality might give an aptamer more protein-like activities, without losing its advantageous nucleic acid-like properties," Famulok explains, "and that might be useful in certain cases where you would like to use an aptamer as a diagnostic or a drug." He also envisions using this approach for the engi- neering of complex, higher order DNA structures-for example, building modified DNA molecules with altered charge distributions that can self-assemble in a manner similar to the way proteins interact. And although the work still remains to be done, the same principles could apply to the functionalization of RNAs, creating new possibilities for the fine regulation of microRNAs. "You could introduce caged bases into a microRNA in an enzymatic fashion," suggests Famulok, "and then try to activate this microRNA by a light pulse, or something like that."

Most of all, Famulok hopes that his group's work will provide a valuable reference for other researchers with innovative ideas: "If people would want to use high-density functionalized DNA for anything - for ideas that I'm too stupid to come up with-then they know how to make them, and they can find very important guidelines in our paper."

\section{Michael Eisenstein}

\section{RESEARCH PAPERS}

J äger, S. et al. A versatile toolbox for variable DNA functionalization at high density. J. Am. Chem. Soc. 127, 15071-15082 (2005).

\section{IMMUNOCHEMISTRY}

\section{A biological tool with chemical applications}

Chemistry researchers demonstrate the utility of the sandwich immunoassay for high-throughput screening of catalysts of organic coupling reactions.

Where would biologists be without the myriad of synthetic methods and small molecules developed by chemists? Unfortunately, the door does not usually swing both ways; biological tools are rarely used to solve purely chemical problems.

Frédéric Taran, of the French Atomic Energy Commission, aims to change this imbalance, by demonstrating that biological assays can in fact have useful chemical applications. "I was fascinated by the analytical potential of immunoassays," he says. "They allow the direct measurement of a specific product in complex mixtures without any work-up, and they are fast, precise and sensitive... everything a chemist needs for analyzing reactions!”

Sandwich immunoassays (known more commonly to biologists as enzyme-linked immunosorbent assays; ELISAs) use two monoclonal antibodies that recognize distinct binding epitopes on a single antigen. One antibody is immobilized on a solid support and the other antibody is conjugated to an enzyme that converts a substrate to an easily detectable product. Taran and colleagues extended the idea to detect the products of bond-forming organic coupling reactions. They raised antibodies to recognize small-molecule tags linked to chemically reactive groups. After allowing the chemical reaction to proceed, they analyzed the crude reaction mixture by ELISA and detected bond formation by monitoring the conversion of the enzyme substrate to a colored product.

As chemists, Taran and colleagues are interested in developing high-throughput screening methods to aid in the discovery of synthetic catalysts, and they show that their sandwich immunoassay can be used as an extremely fast, sensitive and general screen for catalysts that trigger bond formation between reactive species. Notably, many reactions can be screened simultaneously in a microtiter plate, using an automatic plate absorbance reader to quantify the product.

Taran hopes that their screening method will be viewed as a useful tool by industrial chemists. He says, "Many chemists are not familiar with immunoassay techniques and are often convinced that they are complicated to set up. But I think this is not the case; these techniques can be routinely run to answer chemical questions without large investments."

\section{Allison Doerr}

\section{RESEARCH PAPERS}

Vicennati, P. et al. Sandwich immunoassay as a high-throughput screening method for crosscoupling reactions. Angew. Chem. Int. Ed. 44, 6863-6866 (2005) 